\title{
The Status of R\&D on Material Accountancy of Fuel Debris at Fukushima Daiichi Nuclear Power Station
}

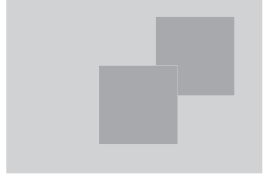

\author{
Japan Atomic Energy Agency, Keiichiro Hori
}

The removal of the molten core fuel from the reactor vessel at the Fukushima Daiichi Nuclear Power Station is scheduled to begin in around 2020. A method of accounting for and controlling nuclear material in the removed fuel debris is being explored in a research and development project to facilitate the decommissioning of the reactors at the nuclear power station. This commentary describes the current status of this project, which is being led by the Japan Atomic Energy Agency and the Tokyo Electric Power Company.

\section{Introduction - Starting Out as Part of a National Decom- missioning Project -}

The Japanese government and the Tokyo Electric Power Company (TEPCO) had been exploring the necessary technologies for decommissioning the Fukushima Daiichi Nuclear Power Station. One of the agenda items to have been identified is the development of a technology for measuring the quantity of nuclear materials in the molten core fuel. In light of this, a project was launched in April 2012 to develop a method of accounting for and controlling nuclear material in the fuel debris.

This commentary describes the policy and current status of this project, which the author has been engaged in since August 2012.

DOI : 10.15669/fukushimainsights.Vol.2.216

(C) 2021 Atomic Energy Society of Japan. All rights reserved.

Originally published in Journal of the Atomic Energy Society of Japan (ISSN 1882-2606), Vol. 57, No. 2, p. 91-93 (2015) in

Japanese. (Japanese version accepted: October 6, 2014) 


\section{Method of Accounting for and Controlling Nuclear Material in the Core Fuel - Statutory Accounting for and Control of Nuclear Material and Declaration of the Inventory of Nuclear Material Pursuant to International Safeguards Agreements with the International Atomic Energy Agency (IAEA) -}

Prior to the recent nuclear accident, the quantity of nuclear materials in the core fuel had been calculated based on the irradiation history of the fuel and information from the fuel fabrication facility. The quantities of uranium and plutonium per fuel assembly had been recorded along with their physical and chemical forms and locations in the facility and other information related to the fuel assembly. The facility operator submitted such records to the Japanese government pursuant to the domestic law. The government of Japan in turn declared this information to the IAEA pursuant to the safeguards agreement between the IAEA and Japan. However, conducting material accounting in units of assemblies will be difficult as it is unlikely that the fuel assemblies at the Fukushima Daiichi Nuclear Power Station retained their original shapes after the accident. For this reason, an alternative method of material accounting needs to be developed.

\section{Role of Nuclear Material Accounting - Changing the Role with the Times -}

Initially, in 1960s and 1970s, nuclear facility operators performed accounting for their nuclear materials with the intention of managing their property and safety. Around the 1990s, however, a gap emerged in Japan between the information needed for property and safety management and that needed for nuclear safeguards. The operators realized that it was less necessary for them to acquire information through material accounting for property and safety management than it was for nuclear safeguards. There were two major causes of the abovementioned gap. Firstly, Japan began demonstrating the peaceful use of plutonium during the same period, which required the implementation of strict nuclear safeguards, thereby resulting in material accounting requiring an extremely high degree of accuracy that went beyond the level required for property and safety management. Secondly, the technological criteria for the IAEA safeguards inspections had been established following considerable discussions, so the information required for the inspections was gradually clarified and increased.

The IAEA safeguards that were established during the 1970s under the comprehensive safeguards agreements form a system in which the state requests the IAEA to certify that the country's nuclear materials are not being diverted from peaceful uses. According to the system's basic principle of minimizing any disruption to peaceful nuclear activities, the system was designed so that safeguards verifications are conducted based on the nuclear facility operator's regular nuclear materials accounting and control.

In light of this historical background, the main purpose of nuclear material accounting is now considered part of safeguards in Japan. In principle, however, safeguards implemented by the IAEA are still supposed to take full advantage of the material accounting conducted by the nuclear facility operator according to their own needs. Moreover, inventory 
management by the facility operator continues to be based on their material accounting and control.

\section{Relationship with IAEA Safeguards - Relationship be- tween the IAEA Safeguards Inspections and Nuclear Material Accounting -}

Under its safeguards system, the IAEA validates the inventory of nuclear materials declared by the governments and directly inspects these materials during on-site inspections of the relevant facilities.

Safeguards inspections employ a technology for detecting the diversion of nuclear materials to ensure that no nuclear materials are diverted to other purposes. As nuclear facilities grow in size, they handle an increasing quantity of nuclear materials. Inspections of facilities that handle nuclear materials in bulk require the use of advanced technologies and various kinds of information to deal with the complex handling processes and limitations in relation to measuring the accuracy of nuclear materials. In other words, the nuclear facility operator is required to provide highly accurate and diverse types of information about nuclear materials to conduct safeguards inspections. Consequently, the costs of safeguards implementation have increased while the operator is required to conduct advanced material accounting.

For this reason, methods have been explored to identify the implementation of reasonable material accounting that can be conducted without causing any disruption to peaceful nuclear activities and obtain highly reliable safeguarding outcomes (annual conclusions regarding any signs of nuclear materials being diverted from peaceful purposes) through the inspections. Along the way, the technical requirements for inspections and material accounting have also gradually changed.

In light of this change, the development of a well-balanced material accounting method befitting the intended inspection method is important for facilitating the operation of nuclear facilities. The same holds true for the development of a method of conducting material accounting of fuel debris.

Under the safeguards system, technically advanced safeguards and inspection methods are applied to plutonium and highly enriched uranium as they are classified as materials that can be directly used in the manufacture of weapons. Material accounting must be explored keeping this in mind.

\section{Management of Nuclear Materials in Light of Past Core Fuel Accidents - Findings from Similar Cases -}

Nuclear material accounting became difficult after the core fuel accidents that occurred at the Three Mile Island Nuclear Power Station (TMI-2) in the United States, the Chernobyl Nuclear Power Plant (ChNPP) in the former USSR, and the Paks Nuclear Power Plant (Paks) in Hungary. In some respects, however, these all differ from the accident in Fukushima. There was no need to declare the quantity of nuclear materials after the accident at TMI-2 as the IAEA safeguards system was not applicable to the United States. Similarly, the former USSR was not subject to IAEA safeguards when the accident took place at ChNPP. 
Safeguards measures were applied after the accident when Ukraine gained its independence from the Soviet Union. This means that the nuclear material inventory in the reactor core had not been declared to the IAEA before the accident. Nevertheless, the inventory of nuclear material after the accident had to be managed and controlled for the application of safeguards. At the Paks nuclear plant, a fuel assembly fell apart when the cladding that contained the fuel pellets broke. Fortunately, all of the fuel pellets could be collected as the fuel pellets did not melt. Studies on nuclear material management at these three plants have yielded the following findings.

- Accounting in relation to removed nuclear materials must be performed in units of containers (TMI-2 and Paks).

- Containment monitoring can be conducted as a safeguarding method to confirm that materials from affected reactors are not being diverted (ChNPP and Paks).

- Some kind of safeguarding method must be applied to containers for removed nuclear materials (ChNPP and Paks).

- There is no precedent for the direct and precise measurement of the quantity of nuclear materials from outside a container. However, the quantity of nuclear materials was once estimated based on the radiation dose measured from outside a container (Paks).

- If the nuclear materials cannot be removed entirely from a reactor, the remaining quantity must be measured or assessed (TMI-2).

\section{Policy on the Development of Technologies for Material Accounting - Development of Practical Management Tech- nology -}

As mentioned earlier, material accounting is essentially intended for the performance of nuclear material management by the nuclear facility operator itself. For this reason, the applied method must basically be practical enough to allow a facility operator to perform material accounting. Accordingly, the basic principle of minimizing obstructions to the main process of decommissioning and completing it in line with a decommissioning roadmap was adopted for the development of all technologies for the method of accounting for and controlling nuclear material in relation to fuel debris.

Importantly, development goals need to be provided in coordination with the regulatory authorities and the IAEA to ensure that no unnecessary goals for safeguards inspections are involved and avoid any overlapping efforts at a later period date.

Given that the technologies for measuring the quantity of nuclear materials can be used for the safeguards verification, development efforts must bear in mind their possible application in safeguards inspections (e.g., enabling inspectors to employ developed technologies). If the developed technologies are employed for safeguards verification, the decommissioning processes can possibly be reduced even further by performing measurements and verifications at the same time. 


\section{Development of Technologies through International Co- operation}

A practical development initiative was commenced through joint research after the signing of an agreement between the Japan Atomic Energy Agency (JAEA) and the National Nuclear Security Administration (NNSA) of the Department of Energy (DOE) of the United States on the safeguard technology for Fukushima Daiichi Nuclear Power Station in November 2012. The JAEA and TEPCO work in tandem to undertake development initiatives in Japan that are centered on this joint research. They also involve the Central Research Institute of Electric Power Industry (CRIEPI) and follow advice provided by the regulatory authorities and the IAEA.

The reasons for the adoption of such an approach are as follows.

Development of material accounting technologies by envisaging safeguarding methods

It is essential that material accounting technologies be developed while keeping in mind the safeguarding methods employed by the IAEA. The JAEA has extensive experience in the development of material accounting and safeguards systems for facilities that handle nuclear materials in bulk. For this reason, the JAEA understands the existing IAEA safeguards system and inspection methods that are employed at actual nuclear facilities. The development of suitable technologies that are practical and reasonable in cooperation with TEPCO is deemed possible.

Application of international findings

The NNSA of the DOE has a long history, extensive experience, and sufficient knowledge in relation to the development of technologies for measuring the quantity of nuclear materials for nuclear safeguards. The NNSA also keeps track of trends in safeguards of the IAEA, and their findings and experience are conducive to the development of suitable measurement technologies for implementing safeguards at the Fukushima Daiichi Nuclear Power Station. They have been working with the JAEA for over 30 years in the development of technologies for safeguards and the like.

Ensuring international transparency

Since the introduction of the Additional Protocol in the 1990s, more effective and streamlined IAEA safeguards have been pursued by, for example, introducing the integrated safeguards approach and the state-level safeguarding approach. One of the nationally led initiatives that have been implemented in response to the updated IAEA safeguards system is the enhancement of transparency in nuclear activities in the state. International collaboration in research and development, the publication of outcomes, and other efforts to enhance transparency in nuclear activities are expected to contribute to IAEA safeguards. The reliability of Japan's safeguards conclusions can probably be enhanced by demonstrating to the world the due consideration that the country has given to nuclear materials in molten fuel with respect to nuclear non-proliferation.

\section{Current Status of the Development of Measurement Tech- nologies}

According to the current decommissioning roadmap (as of October 2014), the removal of fuel debris from Units 1 and 2 at the Fukushima Daiichi Nuclear Power Station is planned for 2020. In line with this roadmap, the development of the necessary method of accounting for 
and controlling nuclear material is underway in phases that last roughly every two years. From 2012 to 2014, studies have been conducted on nuclear material management methods and technologies that have been adopted in similar situations for measuring the quantity of nuclear materials in fuel debris. An evaluation of the feasibility of technologies for measuring the quantity of fuel debris will be conducted from 2014 to 2016, along with an exploration of a system concept for material accounting. The demonstration test (2016-2018) and production of the measurement system (2018-2019) are expected to be completed for the removal of debris in accordance with the decommissioning roadmap.

Nonetheless, the development of the measurement system must keep pace with the development of containers for the removed fuel debris and the removal method. For this reason, the need for a method of accounting for and controlling nuclear material has to be presented to various bodies, such as the group in charge of developing containers, to aid in the pursuit of an efficient design for the debris removal process.

As mentioned earlier, the findings from studies on similar accidents suggest that material accounting must be performed for removed fuel debris in units of containers. In addition, the performance of non-destructive measurements from outside the containers is deemed appropriate for the removed fuel debris to minimize the disruption to other basic decommissioning processes. With these implications in mind, the application of non-destructive measurement technologies for fuel debris in the Fukushima Daiichi Nuclear Power Station was evaluated by experts from the United States and Japan in terms of measurement costs, feasibility, measurement times and other factors that have an impact on the decommissioning process. To date, seven primary measurement technologies and 14 auxiliary measurement technologies have been selected. Basic research and development have been commenced for four of the seven primary technologies.

Going forward, a conceptual design will be developed for specific measurement devices that rely on these technologies, a performance evaluation will be conducted by means of simulations, and then a demonstration will be performed.

\section{Conclusions - Future Direction of the Project -}

The important steps that need to be taken next include the development of a rational method of material accounting that takes into consideration transportation, storage, and other tasks related to the removed fuel debris. As the country that caused the most recent nuclear accident, Japan needs to demonstrate its sincerity to the international community by responsibly managing its nuclear materials.

Moreover, there are various possible ways that measurement technologies can be applied to the nuclear materials in fuel debris whose composition and shapes are hard to determine. We intend to pursue further development initiatives while keeping an eye out for such possibilities. 